\title{
Safety in Chinese Medicine Research
}

\author{
King-Fai Cheng, Ping-Chung Leung* \\ Institute of Chinese Medicine, Prince of Wales Hospital, The Chinese University of Hong Kong, Hong Kong, China \\ Email: *pingcleung@cuhk.edu.hk
}

Received November 14, 2011; revised December 8, 2011; accepted December 22, 2011

\begin{abstract}
Safety of drugs is a common concern, regardless of traditional Chinese medicine or Western medicine. Historical experiences tell us that ignored drug safety evaluation would lead to serious consequences. Although Chinese medicine has been used for thousands of years, re-evaluation of the safety is still very important. The criteria for safety of Chinese herbal medicines should be the same as those for chemical drugs. Many Chinese herbal medicines have a long history of traditional use. However, many of them do not possess proven safety and efficacy by today's standards. Welldesigned randomized controlled trials and comprehensive pre-clinical toxicological studies were not done. Although the lack of such evidence does not absolutely count against the efficacy and safety of Chinese herbs, laboratory and clinical investigations are still needed before the herbs could be considered safe. This paper reviews the evaluation on the safety of Traditional Chinese Medicine.
\end{abstract}

Keywords: Traditional Chinese Medicine; Safety Evaluation; Toxic Chinese Herbs; Adverse Reactions

\section{Introduction}

\section{The Need for Safety Evaluation}

In the last century, more than 40 major drug disasters happened in the world. Such incidents alerted people of all levels about the importance of drug safety. For example, the "thalidomide" event that occurred in the 1960s of the last century might be the most tragic and dramatic drug disaster. Thalidomide was used to treat ailments of pregnant women. Teratogenicity of thalidomide was not considered important. The result was 8000 to 10,000 babies were born with congenital malformations [1-3]. Such disasters caused by chemical drugs made clinicians and authorities realize the importance of drug safety before promotion. In the situations of traditional Chinese medicine, although the history has been over 3000 years, and has been generally considered safe; the safety data derived from scientific tests remain very limited. We do not have essential data of many commonly used traditional Chinese medications such as maximum safe dose and duration of treatment that is considered safe.

In actual fact, many commonly used Chinese herbs are already known to be highly toxic, such as Strychnos, Radix Aconiti, Radix Aconiti Kusnezoffii, Fructus Crotonis, Sheeploitered Azalea etc. Others such as Rhizoma Arisaematis, Rhizoma Pinelliae, Realgar, Rhizoma Polygoni Cuspidati, and Radix Sophorae Tonkinensis are moderately toxic; while many others are mildly toxic. Tradi-

\footnotetext{
*Corresponding author.
}

tionally, the principles of prescription in Chinese medicine include the theories of "nineteen avoidances (十九 畏)” and “eighteen clashes (十八反)” which lay down the recommendations on the avoidance of certain herbs with regard to either solitary use or in combination. Although the toxicity effects of medicinal herbs could be infrequent, slow, and moderate, when compared with chemical drugs, however herbal toxicity could also be more complex, and more obscure.

The compositions of Traditional Chinese medicine are derived not only from plants, but also consist of minerals, and animal products. The degree of toxicity of toxic herbs may be affected by the soil, water, and breeding conditions. All of which are vulnerable to different forms of pollutions like pesticides, fertilizers and other toxic substances. The more over authentications of the herbs could be defective and counterfeit herbs mixed with genuine supplies are not uncommon, thus giving more threat to safety. Processing of medicinal herbs, their storage, and transports, further complicated the safety issue. With regard to proprietary herbal medicinal products, the situation is more complicated. There could be adulterations of chemicals or pharmaceuticals, incompatible combinations, and/ or dangerous dosages. On the whole, due to the limited information available, misunderstanding and negligence tend to be common. Therefore, when using Chinese medicine, we cannot rely only on traditional beliefs from either classical documents or experience. A comprehensive evaluation on the safety of the medicine we intend to use is mandatory on both clinical and research levels. 


\section{Current Status of Safety Evaluation in Chinese Medicine Research}

\subsection{Toxicity Studies Basing on the Traditional Herbal Classification}

As the chemical compositions of medicinal herbs are extremely complex, the study on their toxicology is difficult. The traditional principles of "nineteen avoidances" and "eighteen clashes" could be helpful but are obviously insufficient. There have been no systematic studies on the safety and compatibility of the pairs of herbs referred within the framework of the principles.

The Traditional classification of "toxic Chinese herbs" was mostly based on the ancient clinicians' experiences and records. “Shen Nong Ben Cao Jing” <神農本草經> divided traditional Chinese herbs into toxic and non-toxic types, without reference to their degree of toxicity. “Compendium of Materia Medica” <本草綱目> classified the toxic herbs into four grades i.e. extremely toxic, highly toxic, moderately toxic, and slightly toxic without providing useful objective data to support the classification. Today, the pharmaceutical classification of toxicity is based on experimental data using animals which are fed with the drug until poisoning or death. Toxicity is therefore described quantitatively as the median lethal dose $\left(L D_{50}\right)$. When the oral lethal dose is less than $5 \mathrm{~g} / \mathrm{kg}$, the drug is classified as extremely toxic; the lethal doses between $5-15 \mathrm{~g} / \mathrm{kg}$ and $16-50 \mathrm{~g} / \mathrm{kg}$ are classified as moderately and slightly toxic. Non toxic drug refers to those producing mortality with doses of over $50 \mathrm{~g} / \mathrm{kg}$ (Table 1) [4]. The modern pharmacological concepts of toxicity also include acute toxicity, subacute and chronic toxicity, special toxicity (mutagenicity, teratogenicity, carcinogenicity, abortion, and addiction toxicity etc). None of these modern methods of safety evaluation has been systematically applied in the evaluation of traditional Chinese medicine. The defective guarantee on safety has certainly hindered the globalization of traditional Chinese medicine.

Table 1. Toxic classification of Chinese medicine.

\begin{tabular}{|c|c|c|c|}
\hline Item & Highly toxic & $\begin{array}{l}\text { Moderately } \\
\text { toxic }\end{array}$ & Slightly toxic \\
\hline Symptom & Very severe & Severe & Common side effect \\
\hline Organs damaged & Key organs & Key organs & Rare organ damage \\
\hline High dose & Death & Death & Rare death \\
\hline $\mathrm{LD}_{50}$ (oral) & $<5 \mathrm{~g} / \mathrm{kg}$ & $5-15 \mathrm{~g} / \mathrm{kg}$ & $16-50 \mathrm{~g} / \mathrm{kg}$ \\
\hline $\begin{array}{l}\text { Treat dose } \\
\text { and toxic dose }\end{array}$ & Very close & Close & Not close \\
\hline $\begin{array}{l}\text { Toxic dose } \\
\text { for adult }\end{array}$ & $<3 \mathrm{~g}$ & $3-12 \mathrm{~g}$ & $13-20 \mathrm{~g}$ \\
\hline $\begin{array}{l}\text { Poisoning } \\
\text { incubation }\end{array}$ & $<10 \min$ & $10-30 \mathrm{~min}$ & $\begin{array}{l}>30 \text { min or } \\
\text { accumulation }\end{array}$ \\
\hline
\end{tabular}

Our literature reviews indicated that scholars in the Chinese medicine field have actually done much work on the toxicity of traditional medicinal herbs. Interesting areas include:

1) The study of "eighteen clashes"-the reasons behind incompatible herbs: e.g. toxicity increases when Euphorbia kansui is used with Radix Glycyrrhiza; Adix aconiti lateralis preparata with Rhizoma Bletillae; Veratrum nigrum L. with Schizophyllumcommuneh or White Paeony Root [5]. Efficacy might also be affected because of incompatibility. Modern laboratory studies have shown that Yougui drink with ginseng lengthened the swimming time of mice. However, if Veratrum nigrum L. is added, the effects on swimming rat became worse compared even without ginseng [6].

2) Researches on medicinal herbs containing aristolochic acid were plentiful because of many reports of serious toxicity [7-9].

3) Other areas of extensive research work include: Berberine-induced neonatal jaundice [10], Cinnabar and arsenic contained herbs $[11,12]$ and heavy metals related to medicinal herbs $[13,14]$.

\subsection{Herb-Drug Interactions}

TCM medications could affect the metabolism of chemical drugs which are being taken while their toxicities might also be significantly altered by the chemical drugs [15-18].

Synergistic or additive therapeutic effects may lead to unfavorable adverse events related to over-dosage. On the other hand antagonistic interactions could result in deficient efficacy and therapeutic failure. The potential interaction of herbal medicines with chemical drugs is a major safety concern, which must not be underestimated because the use of herbal medicines is gaining popularity.

A distinguished herbal expert of the Yuan Dynasty (about 1300 AD) Zhang Zhi-he wrote in his collection of clinical cases (儒門事親) [19]. “Toxicity is a property of all drugs not just that poisons are toxic. One must not forget that herbs as popular as Liquorice (Radix Glycyrrhizae) and Radix Sophorae Flavescentis could also be toxic. Undesirable outcomes follow over dosages.” Curative and toxic potentials are properties of all drugs. The key to successful treatment is to select the right drug, the right dosage and the right channel of administration. The simultaneous administration of Chinese medicine and pharmaceuticals must be arranged with care. It has already been reported that when herbal medicine is used together with insulin injection or oral anti-diabetics for diabetic control, hypoglycemia frequently result [20].

The interactions between Chinese herbs and modern drugs should be common issues, yet reports are fewer. Likewise medicinal herbs are getting popular in the US, yet toxicities and interactions related are not thoroughly 
known. According to a survey in the US on 1000 patients attending the accident and emergency departments, 538 of them were using over 1087 types of various forms of drugs, and 30 of them have already shown unfavorable side-effects [21].

Drug interactions could be investigated by in vitro and in vivo experiments, but the results obtained could be inconsistent. For example, St. John's wort, an herb commonly used in America was shown to suppress monoamine oxides in vitro, but such observations could not be demonstrated in vivo studies.

When used appropriately herbal medicine used together with modern medicine may produce better outcomes. For example, in the case of sore throat and tonsillitis, coadministration of Radix Isatidis (板蘭根) with Trimethoprin has been found to significantly strengthen the immune system, which might be responsible for the better outcome.

On the contrary, disastrous outcomes may follow the inappropriate use of Chinese medicine together with modern medicine. For example, co-administration of digoxin and Liu Shen Pill (六神丸) had led to repeated ventricular extrasystoles; and mixing Cinnabaris (硃砂) containing Chinese Medicine with halogen compounds could produce disastrous toxic outcome [22].

Some herbal medicine, apparently safe, could become toxic with overdose. Aloe vera is a good example. In high doses, Aloe vera led to hypersensitive reactions of erythema, urticaria, even nausea and diarrhoea in children; uterine bleeding in pregnant women, and bleeding among those suffering from haemorrhoids or epistaxis [23].

\section{Study of Safety Dosages of Chinese Medicinal Herbs}

Each drug should have its unique proper dose range. However, for Chinese medicinal herbs unlike chemical drugs, they have not been pharmaceutically tested to define its effective dose, maximal dose and toxic dose. Only classic clinical experiences are available. Obviously, unduly increased doses or long administrations could induce adverse reactions. Even herbs believed to be "mild" such as licorice could induce toxic effects if improperly used [24].

For any drug, its toxicity is closely related to its dosage, and when excessive dose is given, even non-toxic drug should produce adverse effects. Cancer drugs are well known toxic agents and yet could be used cleverly for cancer treatment [25]. A Chinese herb Guanmutong, is usually administered in dose of $3-6$ grams. However it readily produces acute renal failure and anaphylactoid purpura while higher doses are used [26]. Tripterygium wilfordii and its preparations are commonly used in China for the treatment of rheumatoid arthritis. However since its therapeutic dose is very close to its toxic dose, it may lead to gastrointestinal bleeding, even visceral necrosis and renal failure [27,28]. Overdose of another medicinal herb, viz. Vietnamese Sophora Root could cause cardiac failure, shock and even death [29,30]. In addition, Proprietor Chinese medicine has also been reported for severe adverse events. For example, cinnabar sedative pills and Tianwangbuxin Dan (both preparations containing cinnabar) when used together, have been reported to be dangerous [31].

The safety duration of administration of Chinese medicine is another current problem in Chinese medicine research. As the desirable efficacy of Chinese medicine is expected to be slow, the duration of administration cannot be short. Hence, the long duration would invite more adverse effects. It is therefore essential to test out the optimal doses and duration of administration in the event of maintaining both effectiveness and safety.

\section{Toxicological Studies}

Toxicology of Chinese medicine includes experimental toxicology, clinical toxicology and toxicokinetics. Toxicity and adverse reactions of traditional Chinese medicine in the clinical application have attracted general concern but remain insufficient.

The misconception that traditional Chinese medicines are "natural drugs" without toxic effects is still widely believed. In fact, some Chinese herbal medicines are not only toxic, but also carcinogenic.

The Virus Section of the China Institute of Medical Science on Prevention has studied 1693 kinds of Chinese herbs and plants, and found that 52 are carcinogenic [32].

According to a report of the Monitoring Center for Adverse Drug Reactions of the Ministry of Health in China, about 20 million people in China died of adverse drug reactions and drug-induced diseases every year [33]. According to a survey in China on the analysis of 382 adverse drug reaction cases in China in 2006, the proportion of adverse reactions induced by Traditional Chinese Medicine was as high as $25.1 \%$, only second to that of induced by antibiotics [34]. Since the chemical composition of chemical medicine is clear, and the research before marketing is well regulated, the mechanisms of adverse reactions are known. On the other hand, the researches of adverse effects and toxicities of traditional Chinese medicine are not well developed, for example, the study of clinical pharmacology, toxicology and drug metabolism of traditional Chinese medicine are started late and developed slowly, many people are lack of knowledge of side effects or adverse effects of traditional Chinese medicine. In fact, the adverse effects of traditional Chinese medicine are not uncommon.

About 90\% traditional Chinese medicine their toxicological data are still unknown, such as their classification, the toxic ingredients, the toxic reactions, their prevention, treatment and rescue etc. 


\subsection{Systematic Study and Safety Evaluation}

Investigators reviewed the reports of toxic and adverse reactions related to TCM from 110 medical journals from 1915 to 1994 . They found that there were totally 6061 cases of adverse reactions reported. Before 1950s only 26 adverse reactions were reported. The number increased in 1960 s to 147 reported in 1970 s to 398 , and in 1980 s to 2217. From the four years of 1991 to 1994 there were already 3273 cases, which sharp rising trend is therefore obvious [35]. Liver and kidney toxicities are related to Chinese medicinal herbs, and systematic studies and safety evaluation, should accordingly set the priorities.

\subsection{Pre-Clinical Safety Evaluation}

Pre-clinical safety evaluation is intended to provide the scientific evidences to predict the toxicity of a new drug through toxicology tests before it is clinically used. The toxipathological examination is to determine the pathological damages in site, extent, nature, including the longterm effects. Most experiments are conducted through animal tests, which include acute toxicity, long-term toxicity, teratogenicity, and carcinogenicity tests etc (Table 2), all of these tests are inseparable from toxicpathology. The longer the testing period, the more important the toxipathological examination. Therefore, the toxipathology is the most important component of preclinical safety evaluation.

\subsubsection{Acute Toxicity Study}

The objective of acute toxicity study is to assess the acute toxicity of a test item when administered as a single dose followed by an observation period of 14 days and thereby obtain information both about hazard assessment and for ranking articles [36].

For chemical drugs, the toxicity can be classified into 6 levels (Table 3).

Table 2. Content of preclinical safety evaluation of traditional Chinese medicine.

\begin{tabular}{c}
\hline Acute toxicity test (oral) \\
\hline Long-term toxicity test (oral) \\
Acute toxicity test (skin) \\
Long-term toxicity test (skin) \\
Skin/Mucosal irritation test \\
Skin sensitization test \\
Genetic toxicity tests \\
Reproductive toxicity test \\
Carcinogenicity test \\
Drug dependence test
\end{tabular}

However, for most herbal medicines, the value of median lethal dose $\left(\mathrm{LD}_{50}\right)$ is not obtainable with practical measures because of the limits on concentration or volume of the medicine, the acute toxicity can be represented by its maximum tolerable dose (MTD).

\subsubsection{Repeated Dose Toxicity Study}

Acute toxicity test does not fully reflect the safety of Chinese medicine. As traditional Chinese medicine usually requires long periods of administration, a long-term toxicity test could better reflect the safety.

The study provided information on the major toxic effects, indicated target organs and the possibility of accumulation, and would provide an estimate of adverse-effects related to certain dose levels which could be used for chronic studies and for establishing safety criteria for human exposure [36].

Long-term toxicity test is one of the key components of the pre-clinical safety evaluation, and it is the fundamental basis for the approval of the new drug for clinical trial.

The purposes of the repeated dose toxicity tests include:

1) To determine whether the test article is suitable for clinical trial;

2) To predict the possible toxicity and the safety range for the human clinical application;

3) To provide an important reference of the initial dose selected for clinical trial;

4) To determine the key observations and reveal preventive measures in clinical trials.

In short, the data of long-term toxicity test supply evidences to determine whether a drug could be further developed.

The duration of long-term toxicology studies would depend on the nature of the herbal preparation and the actual time of clinical trial application. In general, if the herbal medicine is indicated for single dose administration or for repetitive administrations spanning less than a week, the administration period of long-term test should be 2 weeks to a month; if the herbal medicine is for re-

Table 3. Classification of chemical toxicity (oral) from GB 15193.3-94.

\begin{tabular}{cc}
\hline Category & $\mathrm{LD}_{50}(\mathrm{mg} / \mathrm{Kg})$ \\
\hline Extremely toxic & 1.0 or less \\
Highly toxic & $1.0-50.0$ \\
Moderately toxic & $51.0-500.0$ \\
Slightly toxic & $501.0-5000.0$ \\
Practically non-toxic & $5001.0-15,000.0$ \\
Non-toxic & $>15,000$ \\
\hline
\end{tabular}


petitive administration spanning over a week, the test administration term should be 3 - 4 times the period of clinical treatment (the maximal period is 6 months for rodents, 9 months for non-rodents) (Table 4) [36].

Animal species: The test animals include at least two species (including both rodent and non-rodent).

Rodent: White rats are the most commonly used animals.

Non-rodent: Dogs and monkeys are most commonly used animals.

Dose Levels: 3 different dose levels and 1 control group.

Observation:
General observation: physical signs, weights, appearance. Hematology: blood test.

Blood chemistry: livers, kidneys.

Pathology: pathological diagnosis.

There was research reported that some herbs could induce pathological damages if repeated doses were given (Table 5) [37].

Special toxicity studies include:

Mutagenicity Test;

Teratogenicity Test;

Carcinogenicity Test.

Table 4. Clinical treatment course and experimental administration period.

\begin{tabular}{ll}
\hline Clinical treatment course & Experimental administration \\
\hline Single dose or less than 1 week & 2 weeks to a month \\
over a week & $\begin{array}{l}3-4 \text { times of the period of clinical treatment (Not over } 6 \text { months in maximum } \\
\text { for rodents, Not over } 9 \text { months for non-rodents) }\end{array}$ \\
\hline
\end{tabular}

Table 5. 44 herbs that induce pathological damages through long-term toxicity test.

Superficies-resolving herbs (解表藥)＼cjkstart桑葉 (Folium mori)

Heat-clearing herbs (清熱藥)

Expelling phlegm herbs (化痰止咳藥)

Herbs for purgation (瀉下藥)

Herbs for eliminating dampness and diuresis (滲濕利水藥)

Herbs for dispelling pathogenic wind and removing dampness (祛風濕藥)

Herbs for promoting blood circulation and removing blood stasis (活血化瘀藥)

Supplementing and boosting herbs (補益藥)

Anti-helminthic herbs (驅蟲藥)

Anti-cancer herbs (抗腫瘤藥)

Others (其他)
天花粉 (Radix Trichosanthis), 青黛 (Indigo Naturalis),

青蒿 (Artemisia annua L.), 蒲公英 (Herba Taraxaci),

千里光 (Herba Senecionis Scandentis), 半邊蓮 (Lobelia chinensis),

虎杖 (Rhizoma Polygoni Cuspidati), 野菊花 (Chrysanthemum Indici Flos)

半夏 (Pinellia Tuber), 馬兒鈴 (Aristolochia debilis), 啤酒花 (Humulus lupulus)

大黃 (Rheum palmatum L.), 萫麻子 (Semen ricini)

澤瀉 (Rhizoma Alismatis), 木通 (Akebia Stem)

獨活 (Radix Angelicae Biseratae), 秦艽 (Radix gentianae macrophyllae),

蒼耳子 (Fructus Xanthii), 八角楓 (Alangium chinense),

松蘿 (Chinese Usnea), 雷公藤 (Common Threewingnut Root)

莪術 (Rhizoma Curcumae Aeruginosae), 延胡索 (Rhizoma corydalis)

甘草 (Radix Glycyrrhiza), 補骨脂 (Psoralea corylifolia L.),

白術 (Rhizoma Atractylodis Macrocephalae)

苦楝皮 (Cortex meliae), 常山 (Antifeverile Dichroa Root)

長春花 (Herba Catharanthi rosei), 喜樹 (Camptotheca acuminata), 野百合 (Crotalaria sessiliflora L.), 大豬屎豆 (Crotalaria assamica), 龍葵 (Solanum nigrum L.), 三尖杉 (Cephalotaxus fortunei Hook. f.), 斑蟊 (Spanish fly), 山慈菇 (Pleione bulbocodioides)

甜瓜蒂 (Cucumis melo L), 蟾酥 (Venenum bufonis), 棉籽 (Cottonseed), 鉤藤 (Gambir Plant), 銀杏 (Ginkgo biloba),

博落回 (Macleaya cordata), 馬桑 (Coriaria sinica Maxim) 


\subsubsection{Reproductive Toxicity Study}

The objective is to examine whether the test medicine has toxic effects on animal's reproductivity and whether it has teratogenic effects on their offspring.

The herbal drugs intended for contraceptives, preventing miscarriage, prolactin, pregnancy-related drugs, and those affecting fetal development, the reproductive toxicity testing is required.

Local toxicity study:

The objective of local toxicity test is to examine whether local-applied medicines will cause any irritation or allergic reaction, which includes:

Skin irritation;

Skin sensitization;

Eye irritation.

\subsubsection{Drug Dependence Test}

When the medication is used for analgesic and sedativehypnotics, the drug dependency test is required.

\subsection{Clinical Safety Evaluation}

Olsen conducted a review looking at the toxicity of pharmaceuticals observed in humans compared with those earlier observed in experimental animals. This survey included input from 12 pharmaceutical companies with data compiled from 150 compounds. A total of 221 human toxicity (HT) events were reported. The results showed a positive HT concordance rate of $71 \%$ for rodent and non-rodent species. For non-rodents alone the predictive rate was $63 \%$ of HTs and for rodents alone, it was $43 \%$. The results also showed that 94\% HT were first observed in studies of 1 month or less in duration [38]. Most human toxicity could be found in single dose toxicity test. On the whole, $25 \%$ of human toxicity are observed in safety and pharmacology experiments. Using animals China scholars observed embryonic toxicity in animal experiments using Artemisinin. However, in clinical studies pregnant women taking this drug have not produced abnormal babies [39]. In spite of the weak correlation, animal studies are still important. In vivo studies are more reliable than in vitro experiments. This is particularly important for traditional Chinese medicine, because most Chinese herbal medicines are tested with their gross extracts, with unclear chemicals, impurities, and other variable physical factors might all affect the in vitro results. Therefore, toxicology studies of Chinese medicine must be based on in vivo tests, rather than in vitro studies alone.

Herbal poisoning must be differentiated from adverse reactions. Poisoning refers to tissue and organ damages caused by toxic chemicals in the medicinal herb. Adverse reaction is a response to the herbal item which is noxious and unexpected and which occurs at doses normally used in humans for prophylaxis, diagnosis, or therapy of dis- ease or for the modification of physiologic function. Adverse drug reaction is harm directly caused by the drug at normal doses, during normal use. When conducting a clinical trial of Chinese medicine, it is important not only to observe the clinical efficacy, but also to observe the clinical safety. Clinical safety evaluation of traditional Chinese medicine therefore includes toxicity of the drug, adverse reaction monitoring, and the interactions between traditional Chinese medicine and chemical medicine.

\subsection{Adverse Reactions of Chinese Medicine and Post-Marketing Re-Evaluation}

Animal toxicology study has its limitations, but the clinical study alone is unable to find out all toxicities of the drug. As the number of subjects (sample size) for the clinical study of new drug is limited and the inclusion/exclusion criteria are stringent, some adverse reactions cannot be found during the clinical trial period. According to statistical data, if the incidence of adverse reactions is less than ten thousandth, the adverse reactions cannot be found during pre-marketing clinical trials [40]. Therefore, the toxicology studies of Chinese medicine should be extended to post-marketing evaluation i.e. post-marketing adverse events monitoring.

Adverse reactions for herbal administration might be due to the processing of the herbs duration of its administration, contaminations, individual differences, medicine incompatibility, etc.

Most Chinese decoction or proprietary compound consist of a number of herbs, and each herb contains many chemical ingredients. Once adverse reaction occurred, it is most difficult to identify the causes; and the responsable chemical component.

As pre-market clinical trials of Chinese medicine are usually conducted in specific population groups with special age ranges, unique health conditions and the observational times are limited. Observations on adverse effects, mortalities and quality of life would not have universal value. This gives a post-market evaluation extra values.

Toxicology study of the Chinese Medicine should also make use of new knowledge and new techniques of toxicology, which apply to chemical drug development for its healthy development.

\section{Conclusion}

Chinese herbal medicine is the most important part of Traditional Chinese medicine; their safety issues directly affect the clinical efficacy and drug development, as well as its globalization. The reasons that induce adverse reactions of traditional Chinese medicine are complex and divergent, and require systematic studies. Worries about TCM safety have come up many times because of sentimental reports about mass toxicities. It is therefore timely 
to look for practical, systematic ways to ensure TCM safety through proper evaluations, and tests.

\section{Acknowledgements}

The authors would like to acknowledge the Ming Lai Foundation and The International Association of Lions Clubs District 303-Hong Kong and Macau Tam Wah Ching Chinese Medicine Resource Centre for the support given to the Institute of Chinese Medicine, CUHK.

\section{REFERENCES}

[1] W. Lenz, "Thalidomide and Congenital Abnormalities," The Lancet, Vol. 2, 1962, p. 45.

[2] W. Lenz, "A Short History of Thalidomide Embryopathy," Teratology, Vol. 38, No. 3, 1988, pp. 203-215. doi:10.1002/tera.1420380303

[3] B. J. Yuan and Z. Q. Wang, "The Purpose and the Significance of Preclinical Safety Evaluation,” Military Medical Science Press, Beijing, 1997.

[4] L. Y. Xia, "Modern Toxicology of Chinese Medicine," Tianjin Science and Technology Translation and Publishing Corporation, Tianjin, 2005.

[5] J. L. Li, “Animal Experimental Studies of 'Eighteen Clashes' of Chinese Medicinal Herbs,” Journal of Changchun College of Traditional Chinese Medicine, Vol. 2, No. 3, 1988, p. 89.

[6] X. S. Gao, "Eighteen Clashes Research of Chinese Medicinal Herbs,” Chinese Ancient Books Publishing House, Beijing, 1991.

[7] J. Lu, "Studies on the Aristolochic Acid Related Crude Traditional Chinese Medicine,” Drug Standards of China, Vol. 3, No. 2, 2002, pp. 49-50.

[8] L. S. Wang, "Toxic Effects of Aristolochic Acid," Traditional Chinese Drug Research \& Clinical Pharmacology, Vol. 12, No. 6, 2001, pp. 394-395.

[9] G. Z. Jiang and L. Chen, "New Research Progress of Toxicity of Aristolochic Acid in Traditional Chinese Medicine," Chinese Agricultural Science Bulletin, Vol. 24, No. 9, 2008, pp. 84-87.

[10] N. K. Ho, “Traditional Chinese Medicine and Treatment of Neonatal Jaundice,” Singapore Medical Journal, Vol. 37, No. 6, 1996, pp. 645-651.

[11] L. Zhang, S. H. Gao, C. F. Zhou, et al., "Safety Issues of Traditional Chinese Medical Preparations Containing Arsenic Substances: Review Starting from Niuhuang Jiedu Pian (Wan)," China Journal of Chinese Materia Medica, Vol. 31, No. 23, 2006, pp. 2010-2012.

[12] P. Li, "Research Status of Traditional Medicine Cinnabar," West China Journal of Pharmaceutical Sciences, Vol. 25, No. 5, 2010, pp. 622-624.

[13] K. Yang, X. F. Li, Q. H. Li and G. H. Ding, "Overview of Adverse Reactions of Chinese Medicine Containing Heavy Metals and Arsenic," Chinese Journal of Hospital Pharmacy, Vol. 28, No. 4, 2008, pp. 301-304.

[14] D. X. Wang and X. L. Zhu, "Poisoning of Traditional
Chinese Mineral Medicines Containing Heavy Metal: Clinical Presentation and Management,” Adverse Drug Reactions Journal, Vol. 9, No. 1, 2007, pp. 43-45.

[15] R. L. Page and J. D. Lawrence, "Potentiation of Warfarin by Dong Quai,” Pharmacotherapy, Vol. 19, No. 7, 1999, pp. 870-876. doi:10.1592/phco.19.10.870.31558

[16] B. Cheng, C. T. Hung and W. Chiu, "Herbal Medicine and Anaesthesia," Hong Kong Medical Journal, Vol. 8, No. 2, 2002, pp. 123-130.

[17] A. A. Izzo and E. Ernst, "Interactions between Herbal Medicines and Prescribed Drugs: A Systematic Review," Drugs, Vol. 61, No. 15, 2001, pp. 2163-2175. doi:10.2165/00003495-200161150-00002

[18] A. Fugh-Berman, "Herb-Drug Interactions," The Lancet, Vol. 355, No. 9198, 2000, pp. 134-138. doi:10.1016/S0140-6736(99)06457-0

[19] Z. H. Zhang, "Ru Men Shi Qing," The Yuan Dynasty, AD1300.

[20] The American Society of Anesthesiologists, “Anesthesiologists Warn: If You're Taking Herbal Products, Tell Your Doctor before Surgery,” 2001. http://anestit.unipa.it/mirror/asa2/PublicEducation/herbal. html

[21] J. Doucet, P. Chassagne, C. Trivalle, I. Landrin, M. D. Panty, N. Kadri, J. F. Menard and E. Bercoff, “Drug-Drug Interactions Related to Hospital Administrations in Older Adults: A Prospective Study of 1000 Patients," Journal of American Geriatric Society, Vol. 44, No. 8, 1996, pp. 944-948.

[22] X. A. Zhang, "Drug Induced Diseases,” China Medical Technology Publishing House, Beijing, 1997.

[23] D. M. Tan, “Abuse of Aloe Vera,” Science Daily, Vol. 30, 2001.

[24] J. M. Zhang, "Excessive Intake of Licorice can Cause Potassium Loss,” International Journal of Traditional Chinese Medicine, Vol. 14, No. 6, 1992, p. 14.

[25] J. Hu, Ji. Fang, Y. Dong, S. J. Chen and Z. Chen, “Arsenic in Cancer Therapy,” Anticancer Drugs, Vol. 16, No. 2, 2005, pp. 119-127. doi:10.1097/00001813-200502000-00002

[26] Q. L. Li, “One Case of Allergic Purpura Caused by Excessive Aristolochia Manshuriensis Decoction," China Journal of Chinese Materia Medica, Vol. 25, No. 2, 2000, p. 124.

[27] K. B. Bi and Y. L. Jia, "1 Case of Acute Renal Failure Induced by Overdose of Tripterygium Decoction," China Journal of Chinese Materia Medica, Vol. 25, No. 3, 2000, p. 191.

[28] Z. Y. Cau, "Clinical Analysis of 72 Cases of Acute Poisoning after Oral Tripterygium,” Hunan Journal of Traditional Chinese Medicine, Vol. 8, No. 2, 1981, p. 17-19.

[29] J. Z. Wang, "3 Cases of Tonkinensis Poisoning Report," Clinical Medicine, Vol. 8, No. 6, 1988, p. 279.

[30] Y. M. Wen, “One Death Case of Poisoning after Oral Taking Radix Sophorae Tonkinensis," Chinese Journal of Hospital Pharmacy, Vol. 11, No. 6, 1991, p. 271.

[31] G. T. Zhang and A. Y. Zhao, "Improper Combination of 
Proprietary Chinese Medicine in Clinical Practice,” Chinese Journal of Hospital Pharmacy, Vol. 191, No. 10, 1999, p. 621.

[32] "52 Kinds of Hospital Inhibited Plants Which Contain 'Epsteln-Barr Virus Early Antigen-Inducer’ Have Cancerpromoting Effects," Chinese Hospital Architecture \& Equipment, Vol. 4, 2003, p. 11.

[33] W. L. Xue, "From the Safety of Traditional Chinese Medicine to Its Internationalization," Information on Traditional Chinese Medicine, Vol. 22, No. 2, 2005, pp. 6-7.

[34] Y. P. Xiao, X. H. Ren and Y. X. Wu, "Lianyuan City Drug Adverse Reaction Analysis Report," Journal of Clinical and Experimental Medicine, Vol. 6, No. 5, 2007, pp. 172-173.

[35] S. T. Yuan, "Consideration on the Problem of Ascension of the Case Poisoned by Chinese Traditional Medicines," China Journal of Chinese Materia Medica, Vol. 25, No. 1, 2000, p. 56.
[36] Chinese Medicine Council of Hong Kong, "Registration of Proprietary Chinese Medicine (Application Handbook)," 2004.

http://www.cmchk.org.hk/pcm/pdf/reg_handbook_e.pdf

[37] J. Ma, “The Relationship between Drug Toxicology and Other Disciplines," Shanghai National Center for New Drug Safety Evaluation GLP Training Seminars, Chengdu, 6 March 2011.

[38] H. Olson, G. Betton, D. Robjnson, et al., "Concordance of the Toxicity of Pharmaceuticals in Humans and Animals," Regulatory Toxicology and Pharmacology, Vol. 32, No. 1, 2000, pp. 56-67. doi:10.1006/rtph.2000.1399

[39] L. X. Yang, "Toxicology Studies of Artemisinin and Its Derivatives," Journal of Traditional Chinese Medicine, Vol. 1, No. 1, 1982, pp. 3-6.

[40] Center for Evaluation and Research, “Annual Report of 2001 to Nation,” 2001.

http://www.fda.gov/eder/reports/rtn/2001 